\title{
Marathonläufer haben überraschend gesunde Hüft- und Kniegelenke
}

Häufig lange Stecken zu laufen, scheint den Gelenken nicht zu schaden: Marathonläufer leiden nur halb so oft an Arthrose wie der Rest der Bevölkerung.

\begin{abstract}
Laufen mag zwar gesund für Herz- und Kreislauf sein - aber doch nicht für die Gelenke! So lautet jedenfalls ein bekanntes Vorurteil oder auch nur eine beliebte Ausrede, um sich die Mühen eines Waldlaufs zu ersparen. Richtig ist wohl eher das Gegenteil: Nach Resultaten einer US-amerikanischen Untersuchung [1] werden langjährige Marathonläufer nicht mehr, sondern deutlich weniger von Arthrose geplagt als die übrige Bevölkerung (• Abb. 1).

Für ihre Untersuchung haben Orthopäden um Dr. Danielle Ponzia von der Thomas-Jefferson-Universität in Philadelphia Marathonläufer aus 31 Ländern befragt. Sie konnten Fragebögen zu 675 noch aktiven Läufern auswerten. Diese waren im Schnitt 48 Jahre alt, liefen pro Woche knapp $60 \mathrm{~km}$ und beteiligten sich seit rund 20 Jahren an Marathonläufen. Im Schnitt hatten sie bisher 76 Marathons absolviert, der aktivste Läufer brachte es aufüber 1000 Rennen. Sieben der befragten liefen inzwischen mit einer Hüft- oder Kniegelenksprothese.

Immerhin $47 \%$ der Läufer gaben an, dass bei ihnen in den vergangenen zwölf Monaten Hüft- oder Knieschmerzen aufgetreten waren. $35 \%$ kannten Familienangehörige mit Arthrose.
\end{abstract}

Müller, SpringerMedizin.at

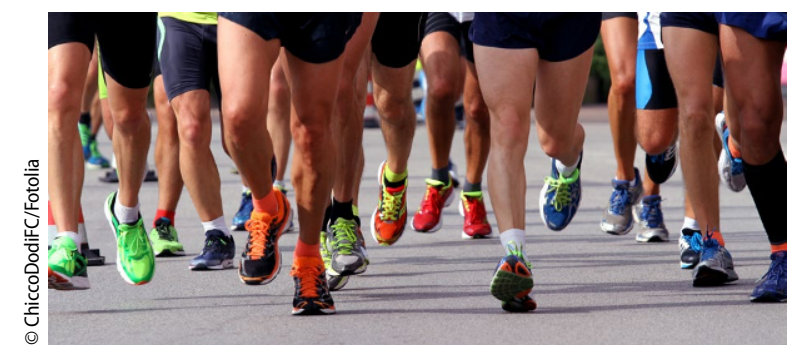

Abb. 1 A Marathonläufer werden deutlich weniger von Arthrose geplagt als die übrige Bevölkerung

\section{Nur $9 \%$ leiden an Arthrose}

Eine Knie- oder Hüftgelenksarthose hatten Ärzte nach Angaben der Läufer bei $8,8 \%$ von ihnen festgestellt. Zum Vergleich: In einem ähnlich alten Ausschnitt der US-Bevölkerung liegt der Anteil mit $17,9 \%$ doppelt so hoch. 5,8 \% der Athleten nannten eine Kniegelenksarthrose, bei 2,1 \% war die Hüfte betroffen, das restliche Prozent klagte über beides.

Wie auch in der übrigen Bevölkerung ist die Arthroserate bei Marathonläufern altersabhängig. Von den über 65-jährigen noch aktiven Läufern war knapp ein Viertel betroffen, in der vergleichbar alten US-Bevölkerung sind es hingegen rund $40 \%$.

Erwartungsgemäß korrelierten Gelenkschmerzen vermehrt mit einer Arthrose, aber auch eine familiäre Belastung erwies sich als Risikofaktor: Diese war bei den Läufern mit Arthrose etwa doppelt so häufig zu beobachten wie bei denen ohne Probleme mit ihrem Bewegungsapparat. Den stärksten Zusammenhang gab es jedoch mit Hüft- oder Kniegelenksoperationen in der Vorgeschichte. Davon waren knapp $13 \%$ betroffen. Von innen hatten wiederum $28 \%$ eine Arthrose. Nach den Berechnungen der Forscher um Ponzia ist das Arthroserisiko für Läufer mit Hüft- oder Knie-Operationen um mehr als das Fünffache erhöht.

Die Gesamtzahl der absolvierten Marathons und das wöchentliche Trainingspensum korrelierten hingegen nicht mit der Arthroserate, sofern Alter und andere Faktoren berücksichtigt wurden.

Zumindest nach diesen Daten scheinen Langstreckenläufe also eher vor Arthrose zu schützen, was sich damit erklären lässt, dass Läufer mehr

\section{Das Wichtigste in Kürze}

Schaden Langstreckenläufe den Gelenken?

Aktive Marathonläufer leiden deutlich seltener an Arthrose als die übrige Bevölkerung.

Bedeutung:Viel zu laufen könnte die Gelenke schützen.

Einschränkung: Daten basieren auf Umfrage bei aktiven Läufern - hier ist keine hohe Arthroserate zu erwarten.

Muskel-, Knochen- und Knorpelmasse ausbilden. Andere Studien deuteten zudem auf antientzündliche und chondroprotektive Effekte von Langstreckenläufen, erläutern die Forscher.

Da die Studie auf freiwilligen Angaben von aktiven Läufern beruht, kann natürlich ein Selektions- und Survivalbias nicht ausgeschlossen werden:Wer aufgrund einer Arthrose nicht mehr laufen konnte, wurde nicht berücksichtigt. Umgekehrt ist auch nicht zu erwarten, dass sich unter den aktiven Marathonläufern viele mit einer ausgeprägten Arthrose befinden. Die Studienresultate sind also mit einer gewissen Vorsicht zu interpretieren. Sie müssten nun anhand prospektiver Untersuchungen bestätigt werden.

\section{Literatur}

1. Ponzio DY et al (2018) Low Prevalence of Hip and Knee Arthritis in Active Marathon Runners. J Bone Joint Surg Am 100(2):131-137. https://doi.org/10.2106/JBJS.16.01071

rheuma plus $2018 \cdot 17: 154$ https://doi.org/10.1007/s12688018-0209-8

(c) Springer-Verlag GmbH Austria, ein Teil von Springer Nature 2018 